\title{
Small Range High Precision Positioning Algorithm Based on Improved Sinc Interpolation
}

\author{
Zhengping Li, Chaoliang Qin, Yongmei Zhang, Li Ma, and Changliu Niu
}

\begin{abstract}
This paper designed an improved positioning system which employed a proposed improved Sinc interpolation algorithm to reduce the sampling frequency of the system , calculated time difference of arrival (TODA) values with matched filter, estimated the position of the target node by Chan's algorithm and used Chauvenet criterion to optimize the positioning results. Analysis and simulation results showed that the algorithm could reduce the sampling frequency to a great extent while ensuring the accuracy of the positioning system.
\end{abstract}

Index Terms-Wireless positioning, TDOA, Chan's algorithm, Sinc interpolation, Chauvenet criterion.

\section{INTRODUCTION}

Small range high precision positioning has broad application prospects. It can be used for monitoring and tracking intensive care, preventing the baby stolen in delivery room and controlling the expensive medical equipment in the medical industry. Small range high precision positioning is urgently needed in interactive games.

The existing positioning technologies cannot meet the requirements of the forementioned applications. The GPS satellite positioning accuracy can be very high, but when the GPS receivers are indoor, the signal strength is greatly attenuated because of the buildings and objects [1]. Some companies have developed small range positioning devices, for example, Xsens proposed MVN MotionGrid for small range positioning and the accuracy can reach $10 \mathrm{~cm}$. But the employed positioning algorithms aren't open for research. Time difference of arrival (TDOA) can be used for high precise positioning system, but few researches have been don't on how to employ TDOA to enhance the precision [2].

This paper designed an improved positioning system which employed the proposed improved Sinc interpolation algorithm to reduce the sampling frequency of the system for TDOA value. Then the system estimated the position of the target node by Chan's algorithm and used Chauvenet criterion to optimize the positioning results. As the result, we can reduce the sampling frequency to the maximum extent on the premise of ensuring the accuracy.

The rest of the paper is organized as follows. Section II made a survey of the related researches, Section III described the related the principle of the proposed algorithm, Section IV made simulation and analysis of the algorithm, and

Manuscript received December 12, 2015; revised March 2, 2016.

The authors are with North China University of Technology, China (e-mail: zhplee@gmail.com).
Section V drew a conclusion.

\section{RELATED RESEARCH}

The concept of wireless location system has been proposed as early as 30 years ago. With the development of technology and the increasing demand of positioning, wireless location technology has made great progress [3].

At present, the positioning method based on distance measurement is mainly based on the distance estimation between nodes to locate the unknown nodes. Distance estimation was done by measuring the distance or angle of the input signal. The existing techniques include: the received signal strength (RSSI), time of arrival (TOA), time difference of arrival (TDOA) etc. We mainly talk about the TDOA techniques.

TDOA technology estimates the location of the target node by detecting the time difference of signal arriving differences among reference nodes. The time difference of the same signal to the two reference nodes determines the hyperbolic of a target node, the location of the target node two can be gotten with two hyperbolic tracks [4]. According to the principle of TDOA positioning, if we can accurately get the time difference of the arrival, we can calculate the exact location of the target node, so the accurate estimation of signal arrival time difference of different reference nodes is the key of the TDOA positioning. Based on the TDOA and the correlation technologies, Zhengping Li and Ziren Wang (2013) put forward a new algorithm that can greatly improve the positioning accuracy. Their system employs matched filter to calculate TDOA value and does not need precise synchronization between the transmitter and receivers so that it makes the TDOA value more accurate. Their positioning accuracy can be less than $1 \mathrm{~cm}$ [5]. The problem is that the positioning gets higher accuracy when the sampling frequency is $10 \mathrm{GHz}$. It is difficult to realize in the actual system. Based on that, this paper proposed an improved positioning system to reduce the sampling frequency in the premise of ensuring the positioning accuracy.

\section{Related Algorithm And the Positioning Process}

In the paper Precision Wireless Positioning Scheme in Small Range Based on First-Order Difference and Correlation Inspection. Their algorithm process is as follows:

In the receiver, the system first did amplitude normalization processing of received FM wave. Then it did match filtering between the received signal after amplitude normalization processing and the match filter signal which was similar to the modulated signal of the transmitter. The 
result shows in Fig. 1.

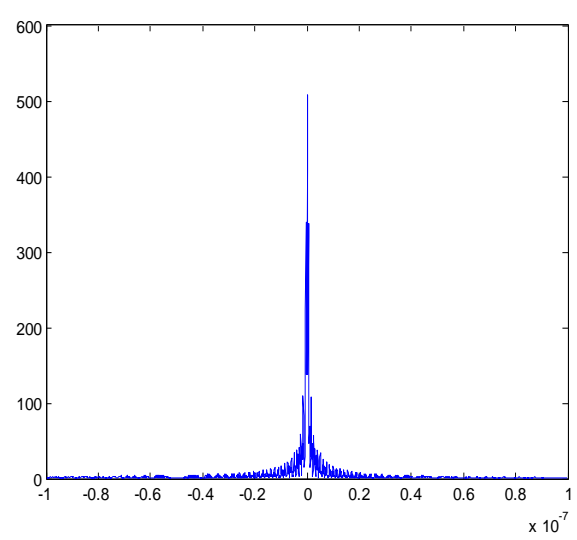

Fig. 1. Matched filtering output signal.

A matched filter input signal was $s(t)$, the impactresponse was $h(t)=s\left(t_{0}-t\right)$, the output of the matched filter was equal to the convolution of the input signal and the impact response:

$$
s_{\text {out }}(t)=s(t) \otimes h(t)=\int_{-\infty}^{\infty} s(\tau) h(t-\tau) d \tau=k R_{s}\left(t_{0}-t\right)
$$

Formula (1) showed that the output signal of matched filter and the autocorrelation function of linear FM signal were the same in form, just had differs of a constant factor $k$ and a time delay $t_{0}$. The autocorrelation function, $R_{S}\left(t-t_{0}\right)$ 's maximum is $R_{S}(0)$, so the output signal of matched filter get the maximum when $t=t_{0}$.

But their problem was that the matched filtering output signal gets a good result when the sampling frequency is $10 \mathrm{GHz}$. It is difficult to realize in the actual system. Based on that, we added the improved Sinc algorithm before the matched filtering process to reduce the sampling frequency.

From results of the matched filtering output signal with improved Sinc algorithm shown in Fig. 2 and Fig. 3, it could be seen that the peak of the matched filter result was also quite obvious. But compared to Fig. 1, there were multiple peaks in the Fig. 2 and Fig. 3. The multiple peaks could have a certain degree of influence on the positioning accuracy but not great. In the fourth section, system simulation and analysis, we would see the concrete results.

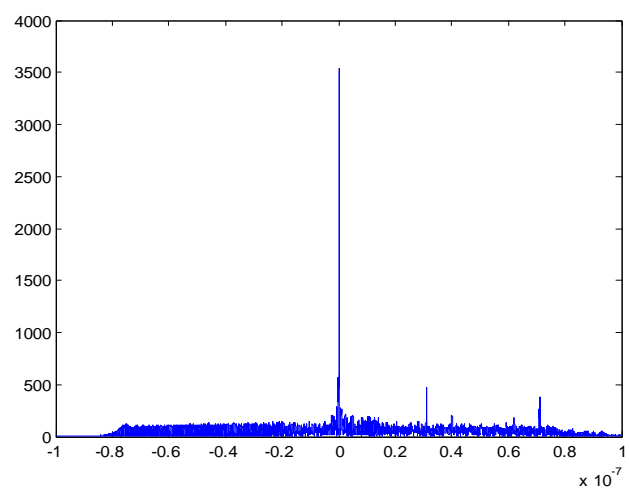

Fig. 2. Matched filtering output signal with improved Sinc algorithm-1GHz.

Because the received signal had a time delay compared with the matched filter signal, the output signal could be gained by doing matched filtering between these two signals. When the output got the maximum, the time was $t_{0}$. A group of different $t_{0}$ values could be gotten by doing matched filtering between the modulation signal and the received signal of each receiver. Suppose that $t_{0}$ of receiver 1 was $T_{1}$, the $t_{0}$ of receiver $i$ was $T_{i}$. Then the TDOA value was $\tau_{i, 1}=T_{i}-T_{1}$. After getting a group of TDOA values, the position of target node could be estimated by Chan's algorithm. The Chan's algorithm was introduced in detail in the paper Precision Wireless Positioning Scheme in Small Range Based on First-Order Difference and Correlation Inspection, so we needn't repeat the algorithm here.

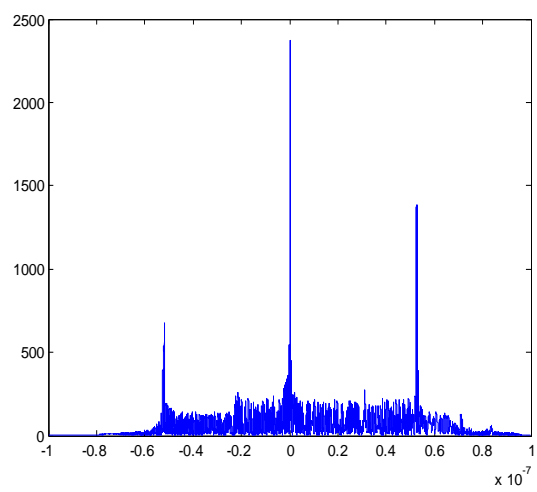

Fig. 3. Matched filtering output signal with improved Sinc algorithm-500MHz.

\section{A. The Positioning Process}

This paper presented a high accuracy positioning method in small range based on an improved Sinc interpolation algorithm, the steps are as follows:

Step 1: $L$ fixed target nodes received the FM wave signals from the reference node, of which $L$ was a positive integer. The modulation signal of the FM wave was sawtooth signal, and so the FM wave was called sawtooth FM wave. A cycle of the sawtooth FM signal was called a chirp;

Step 2: We conducted amplitude limitation on the received signals, sampled on $\mathrm{M}$ continuous chirps at interval $T$ and achieved the sample function $x_{i}(n)$ in which $i=1,2, \ldots, M$; $n=0,1, \ldots, N$. $N$ was the sample point number of each chirp.

Step 3: By using improved Sinc interpolation algorithm to reconstruct the sample function,we could get the reconstruction function $y(k)$ of which $k=0,1, \ldots,(N-1)(M+1)$ $+M$.

Step 4: Sampling the original sawtooth FM waves at interval $T /(M+1)$ and achieving the sample function $u(k)$ where $k=0,1, \ldots,(N-1)(M+1)+M$. Then, we took $u(k)$ and $y(k)$ to perform the cross-correlation operation and obtained the correlation peak location $A_{i}$ of which $i=0,1, \ldots$, L.

Step 5: Using correlation peak location gap we could calculate the signal arrival time difference $t_{2,1}, t_{3,1}, \cdots, t_{i, 1}, \cdots t_{L, 1}$ between the $2^{\text {th }}, 3^{\text {th }}, 4^{\text {th }}, \cdots, L^{\text {th }}$ 
reference node and the $1^{\text {th }}$ reference node. Among them $t_{2,1}, t_{3,1}, \cdots, t_{i, 1}, \cdots t_{L, 1}$ were the TDOA values of which $t_{i, 1}=\left(A_{i}-A_{1}\right) * T . T$ was the time interval of the sample points in $y(k)$;

Step 6: The TDOA values and the coordinates of the reference nodes was put into Chan algorithm to calculate the position of the target node.

Step 7: Chauvenet criterion was used to optimize the positioning results.

\section{B. The Improved Sinc Interpolation Algorithm}

In Step 3 an improved Sinc interpolation algorithm was mentioned, and a detail description of the improved Sinc interpolation algorithm was present in this section.

We used the Sinc interpolation algorithm to reconstruct the sample function $x_{i}(n)(i=1,2, \ldots, M ; n=0,1, \ldots$, $(N-1)(M+1)+M)$ obtained from the Step 2,.

$M$ points were inserted between the two neighboring sampling points and form a new sequence $c_{i}(m, n)(i, m=1$, $2, \ldots, M ; n=0,1, \ldots, N-1)$ from following formula.

$$
c_{i}(m, n)=\sum_{l=0}^{N-1} x_{i}(l) \frac{\sin [(n+m /(M+1)-l) \pi]}{(n+m /(M+1)-l) \pi}
$$

We combine $x_{i}(n)$ and the $M$ points inserted into the sampling points to obtain the reconstructed sequence $y_{i}(h)$ :

$$
\begin{aligned}
& y_{i}(0)=x_{i}(0), \\
& y_{i}(M+1)=x_{i}(1), \quad \cdots, \\
& y_{i}((N-1)(M+1))=x_{i}(N-1), \\
& y_{i}(1)=c_{1}(i, 0), \\
& y_{i}(M+2)=c_{1}(i, 1), \quad \cdots, \\
& y_{i}((N-1)(M+1)+1)=c_{1}(i, N-1), \\
& \quad \vdots \\
& y_{i}(M)=c_{M}(i, M), \\
& y_{i}(2 M+1)=c_{M}(i, M), \cdots, \\
& y_{i}((N-1)(M+1)+M)=c_{M}(i, N-1) ;
\end{aligned}
$$

Namely:

$$
\begin{aligned}
& y_{i}(h)= \begin{cases}x_{i}(n) & , h=n(M+1) \\
c_{i}(m, n) & , h=n(M+1)+i\end{cases} \\
& i, m=1,2, \cdots, M \\
& n=0,1,2, \cdots, N-1 \\
& h=0,1,2, \cdots,(N-1)(M+1)+M
\end{aligned}
$$

$y_{1}(h), y_{2}(h), y_{3}(h), \cdots, y_{M}(h)$ were used to reconstruct again and we obtained the reconstruct sequence $y(k):$

$y(0)=y_{1}(0)$,

$y(M+1)=y_{1}(M+1), \cdots$,

$y((N-1)(M+1))=y_{1}((N-1)(M+1))$,

$y(1)=y_{1}(1)$

$y(M+2)=y_{1}(M+2), \cdots$,

$y((N-1)(M+1)+1)=y_{1}((N-1)(M+1)+1)$,

$y(2)=y_{2}(2)$,

$y(M+3)=y_{2}(M+3), \cdots$,

$y((N-1)(M+1)+2)=y_{2}((N-1)(M+1)+2)$,

$y(i)=y_{i}(i)$

$y(M+1+i)=y_{i}(M+1+i), \cdots$,

$y((N-1)(M+1)+i)=y_{i}((N-1)(M+1)+i)$

$y(M)=y_{M}(M)$,

$y(2 M+1)=y_{M}(2 M+1), \cdots$,

$y((N-1)(M+1)+M)=y_{M}((N-1)(M+1)+M) ;$

Namely:

$$
\begin{aligned}
& y(k)=\left\{\begin{array}{l}
y_{1}(n(M+1)), k=n(M+1) \\
y_{i}(n(M+1)+i), k=n(M+1)+i
\end{array}\right. \\
& n=0,1,2 \cdots N-1, i=1,2,3, \cdots, M
\end{aligned}
$$

Fig. 4. showed the reconstructed sequence $y(k)$.

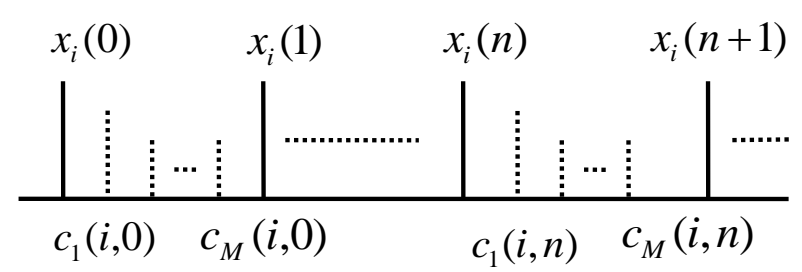

Fig. 4. The reconstructed sequence $y(k)$ with the improved Sinc interpolation algorithm.

\section{Data Processing}

In the results of positioning, multipath signal interference would make some TDOA values greatly deviating from the distance between the target node and the reference nodes. We called these TDOA values the abnormal data. If the abnormal data was used to calculate the target node's position, the positioning error would be very large. Therefore we should eliminate the abnormal data to increase the positioning error.

In the paper Precision Wireless Positioning Scheme in Small Range Based on First-Order Difference and Correlation Inspection, they employ the first-order difference method. The first-order difference method is very suitable for the real time data acquisition and processing 
system. But the precision of the first-order difference method is also related with the measurement precision of the first two values, except the size of the error window. If the measurement is not monotonic increasing or decreasing function, this method will produce large errors at the inflection point.

Chauvenet criterion is also known as the equal probability principle, it is a very common criterion to eliminate error in actual project.

In this system, in Step 7 we used the Chauvenet criterion to search for the values of large error and take them out.

Chauvenet criterion was as follows: During the $n^{\text {th }}$ measurement, we took the number that might not occur for 0.5 , so for the normal distribution, the probability that the error never appear was:

$$
1-\frac{1}{2 \pi} \int_{-\omega_{n}}^{\omega_{n}} \exp \left(-\frac{x^{2}}{2}\right) d x=\frac{1}{2 n}
$$

According to the known value $n$ located in the right side of the equation, we could use the table of the standard normal function to find out the Chauvenet coefficient $\omega_{n}$ For the measured value $x_{i}$, if the absolute value of the difference between the measured value and the average was greater than the product between the standard deviation and Chauvenet coefficient, the measured value should be eliminated.

That was $\left|x_{i}-\bar{x}\right|>\omega_{n} S_{x}$, where $S_{x}$ was the standard deviation.

\section{SYSTEM SIMULATION AND ANALYSIS}

In the simulation system, the positioning area was determined by the number of reference nodes, and the more number of reference nodes the larger of positioning area. The reference nodes were stationary and they should be distributed around the positioning area uniformly as much as possible so that the system could get better positioning results. If the reference nodes were changed to distribute at random or in a straight line in the positioning area, the positioning result would not be as well as before.

In this simulation system, it supposes that the positioning range is $20 \mathrm{~m} \times 20 \mathrm{~m}$. The coordinates of seven reference nodes were $(0,0),(0,20),(10,-4),(20,0),(10,24),(20,20)$, $(-4,10)$. Target node was act as transmitter. The modulation signal's frequency of target node was $1 \mathrm{MHz}$. The simulation supposes that the transmission channel was 6 paths Rician channel which had one line-of-sight (LOS) path and 5 reflection paths. The reflection paths were caused by the multipath effect because of the signal reflection, diffraction and scattering. The additional delay of 6 paths were [0 31/1e9 71/1e9 109/1e9 173/1e9 251/1e9] (s); the additional

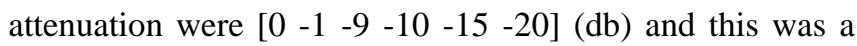
common indoor channel. In addition, the received signal summed together of the LOS signal and reflection signals. If the obstacles were on the LOS path, it sould affect the TDOA value and cause TDOA errors.

The positioning accuracy was measured with the root mean square error (RMSE) of positioning results, which was frequently-used at present. The positioning system simulation was done in different conditions through Matlab:

1) The positioning accuracy with different Sinc interpolation algorithm was shown in Fig. 5.

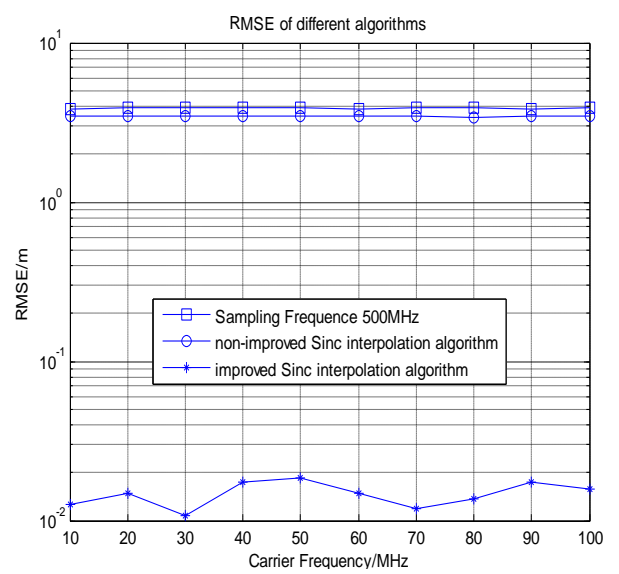

Fig. 5. RMSE of different Sinc interpolation algorithm.

TABLE I: RMSE OF DIFFERENT SINC INTERPOLATION ALGORITHM

\begin{tabular}{|l|l|l|l|}
\hline $\begin{array}{r}\text { RMSE/m } \\
\text { CF/MHz }\end{array}$ & $\begin{array}{l}\text { RMSE } \\
\text { square }\end{array}$ & $\begin{array}{l}\text { RMSE } \\
\text { circle }\end{array}$ & $\begin{array}{l}\text { RMSE } \\
\text { asterisk }\end{array}$ \\
\hline 10 & 3.8295 & 3.4723 & 0.0125 \\
\hline 20 & 3.8724 & 3.4449 & 0.0148 \\
\hline 30 & 3.8928 & 3.4613 & 0.0107 \\
\hline 40 & 3.8995 & 3.4598 & 0.0176 \\
\hline 50 & 3.8983 & 3.4576 & 0.0184 \\
\hline 60 & 3.8196 & 3.4326 & 0.0148 \\
\hline 70 & 3.8812 & 3.4671 & 0.0119 \\
\hline 80 & 3.8886 & 3.4096 & 0.0138 \\
\hline 90 & 3.8700 & 3.4400 & 0.0174 \\
\hline 100 & 3.8979 & 3.4813 & 0.0157 \\
\hline
\end{tabular}

In Fig. 5, abscissa was carrier frequency and the values were $10 \mathrm{MHz}, 20 \mathrm{MHz}, 30 \mathrm{MHz}, 40 \mathrm{MHz}, 50 \mathrm{MHz}, 60 \mathrm{MHz}$, $70 \mathrm{MHz}, 80 \mathrm{MHz}, 90 \mathrm{MHz}$ and $100 \mathrm{MHz}$. Ordinate was RMSE . Table I lists the details of each point in the Fig. 5.The non-improved Sinc interpolation algorithm referred that reference node receives each chirp and then interpolate points behind every sampling points of the sampling chirp [6]. We assumed every sampling chirp contains 1000 point and the interpolation points are 9. So the interpolated chirp contained 10000 points. Finally we used the interpolated chirp to do correlation detection with the frequency-modulated wave that the target node transmitted and retrieved the position of the correlation peak to calculate the TDOA values. The improved Sinc interpolation algorithm referred that the reference node received a chirp and then interpolate points behind every sampling points of the received chirp. But this time we took out some points from 9 successive chirps to form a new chirp to do the correlation detection. The point taking out rules: We regarded the $1^{\text {th }}$ sampling chirp (totally 1000 points) as the basis. Because we had interpolated 9 points behind every 
sampling point, every interpolated chirp has 10000 points. We took out the $2^{\text {nd }}, 12^{\text {th }}, 22^{\text {th }}, \ldots, 9992^{\text {th }}$ interpolation point of the $1^{\text {st }}$ chirp; the $3^{\text {rd }}, 13^{\text {th }}, 23^{\text {th }}, \ldots, 9993^{\text {th }}$ interpolation point of the $2^{\text {rd }}$ chirp; ... ; the $10^{\text {th }}, 20^{\text {th }}, \ldots$, $10000^{\text {th }}$ interpolation point of the $9^{\text {th }}$ chirp. We totally take out 9000 points and then we interpolated the 9000 point to the basis (namely the $1^{\text {th }}$ chirp) to form the new chirp to do the correlation detection.

The sampling frequency of three algorithms in the Fig. 2 was $500 \mathrm{MHz}$. From the simulation results it could be seen that when we used the non-improved Sinc interpolation algorithm, the positioning accuracy had not been significantly improved. The RMSE only decreased from about $3.8 \mathrm{~m}$ to about $3.4 \mathrm{~m}$. But the improved algorithm, comparing with the non-improved algorithm in positioning accuracy, had a very large enhancement. The RMSE only decreases from about $3.8 \mathrm{~m}$ to about $0.01 \mathrm{~m}$.

2) The positioning accuracy in different sampling frequency and interpolation points were shown in Fig. 6:

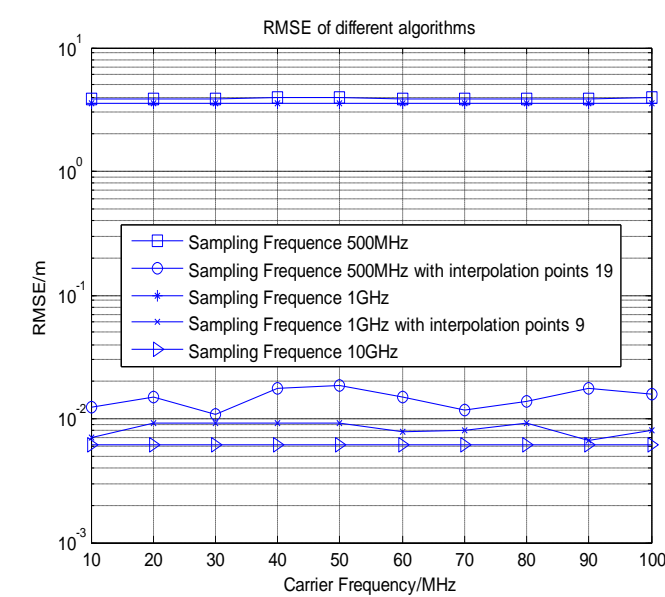

Fig. 6. RMSE of different algorithm in different sampling frequency and interpolation points.

TABLE II: RMSE OF DIFFERENT ALGORITHM IN DIFFERENT SAMPLING FREQUENCY AND INTERPOLATION POINTS

\begin{tabular}{|l|l|l|l|l|l|}
\hline $\begin{array}{c}\text { RMSE/ } \\
\mathrm{CF} / \\
\mathrm{MHz}\end{array}$ & $\begin{array}{l}\text { RMSE } \\
\text { square }\end{array}$ & $\begin{array}{l}\text { RMSE } \\
\text { circle }\end{array}$ & $\begin{array}{l}\text { RMSE } \\
\text { asterisk }\end{array}$ & $\begin{array}{l}\text { RMSE } \\
\text { cross }\end{array}$ & $\begin{array}{l}\text { RMSE } \\
\text { triangle }\end{array}$ \\
\hline 10 & 3.8295 & 0.0125 & 3.5876 & 0.0071 & 0.0062 \\
\hline 20 & 3.8724 & 0.0148 & 3.5935 & 0.0092 & 0.0062 \\
\hline 30 & 3.8928 & 0.0107 & 3.5495 & 0.0092 & 0.0062 \\
\hline 40 & 3.8995 & 0.0176 & 3.5780 & 0.0092 & 0.0062 \\
\hline 50 & 3.8983 & 0.0184 & 3.5430 & 0.0092 & 0.0062 \\
\hline 60 & 3.8196 & 0.0148 & 3.5104 & 0.0079 & 0.0062 \\
\hline 70 & 3.8812 & 0.0119 & 3.5661 & 0.0080 & 0.0062 \\
\hline 80 & 3.8886 & 0.0138 & 3.5749 & 0.0092 & 0.0062 \\
\hline 90 & 3.8700 & 0.0174 & 3.5041 & 0.0067 & 0.0062 \\
\hline 100 & 3.8979 & 0.0157 & 3.5107 & 0.0081 & 0.0062 \\
\hline
\end{tabular}

In Fig. 6, abscissa was carrier frequency and the values were $10 \mathrm{MHz}, 20 \mathrm{MHz}, 30 \mathrm{MHz}, 40 \mathrm{MHz}, 50 \mathrm{MHz}, 60 \mathrm{MHz}$, $70 \mathrm{MHz}, 80 \mathrm{MHz}, 90 \mathrm{MHz}$ and $100 \mathrm{MHz}$. Ordinate was RMSE.
Table II lists the details of each point in the Fig. 6. The target node was stationary and it could be at anyplace in the positioning area. From the simulation results it could be seen that the algorithm with improved Sinc interpolation comparing with algorithm without improved Sinc interpolation in positioning accuracy had a very large enhancement. When the sampling frequency was reduced from $10 \mathrm{GHz}$ to $1 \mathrm{GHz}$ and $500 \mathrm{MHz}$ without improved Sinc interpolation algorithm, positioning accuracy was obviously declined. When the sampling frequency was $10 \mathrm{GHz}$, the RMSE value was quite low but the sampling frequency was $10 \mathrm{GHz}$ which was too high to achieve in practical application. So we reduced the sampling frequency to $1 \mathrm{GHz} / 500 \mathrm{MHz}$ and used the improved Sinc interpolation algorithm to interpolate 9/19 points to the sampling chirps as far as possible to achieve the $10 \mathrm{GHz}$ sampling frequency accuracy. The results showed that the accuracy of the sampling frequency $1 \mathrm{GHz} / 500 \mathrm{MHz}$ with interpolation point $9 / 19$ was very close to the sampling frequency $10 \mathrm{GHz}$. We could also see from results that the carrier frequency had little influence on the positioning accuracy when the carrier frequency varying from $10 \mathrm{MHz}$ to $100 \mathrm{MHz}$.

3) The positioning accuracy in different numbers of interpolation points were shown in Fig. 7:

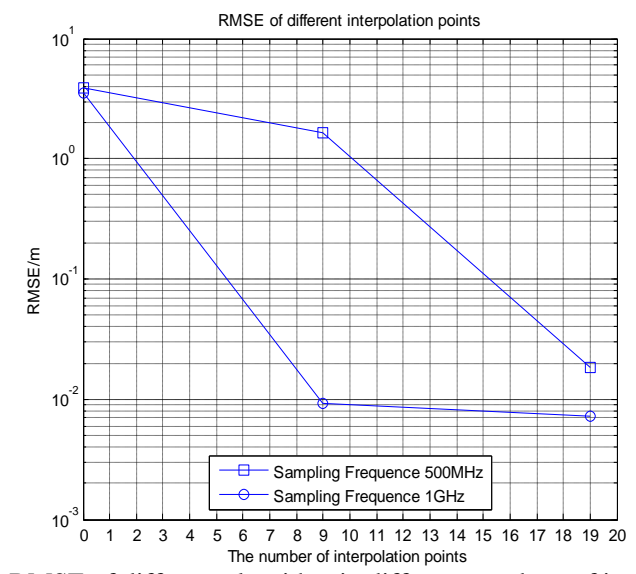

Fig. 7. RMSE of different algorithm in different numbers of interpolation points.

TABLE III: RMSE OF DIFFERENT ALGORITHMS IN DIFFERENT NUMBERS OF INTERPOLATION POINTS

\begin{tabular}{|l|l|l|l|}
\hline RMSE/m & & \\
Interpolation \\
Numpers
\end{tabular}

In Fig. 7, abscissa was the numbers of interpolation points and the values were $0,9,19$. Ordinate was RMSE. We set the carrier frequency to $50 \mathrm{MHz}$. Table III lists the details of each point in the Fig. 7. From the simulation results it could be seen that when there were no interpolation points, the positioning accuracy was low. When we interpolated 9 points to the $500 \mathrm{MHz}$ sampling chirps the positioning accuracy was better than the $1 \mathrm{GHz}$ sampling frequency without interpolation points. When we interpolated $19 / 9$ points to the 
$500 \mathrm{MHz} / 1 \mathrm{GHz}$ sampling chirps, the positioning accuracy improved obviously. Since the positioning accuracy of the sampling frequency with 9 interpolation points was enough high, there was little accuracy improvement when we interpolated 19 points to the $1 \mathrm{GHz}$ sampling chirps .

4) The positioning accuracy in different sampling frequency were shown in Fig. 8:

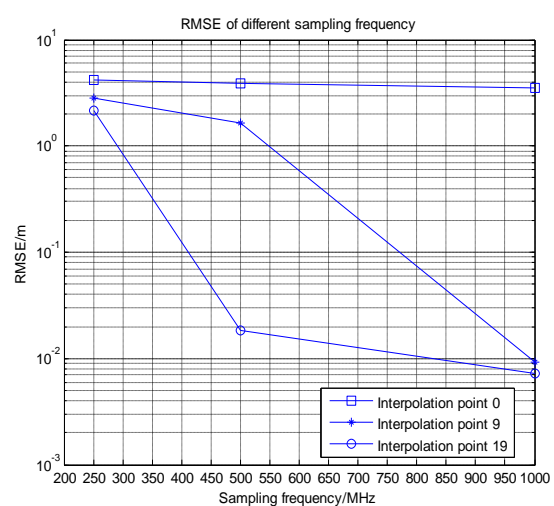

Fig. 8. RMSE of different algorithm in different numbers of interpolation points.

TABLE IV: RMSE OF DIFFERENT ALGORITHMS IN DIFFERENT SAMPLING

\begin{tabular}{|l|l|l|l|}
\hline \multicolumn{5}{|c|}{ FREQUENCY } & \\
RMSE/m & & & \\
& & 500 & 1000 \\
\hline RMSE(square) & 250 & & \\
\hline RMSE (circle) & 4.1463 & 3.8983 & 3.5430 \\
\hline RMSE (asterisk) & 2.8120 & 1.6520 & 0.0092 \\
\hline
\end{tabular}

In Fig. 8, abscissa was the sampling frequency and the values were $250 \mathrm{MHz}, 500 \mathrm{MHz}, 1000 \mathrm{MHz}(1 \mathrm{GHz})$. Ordinate was RMSE (Unit m). We set the carrier frequency to $50 \mathrm{MHz}$. Table IV lists the details of each point in the Fig. 8. From the simulation results it could be seen that the positioning accuracy was not high in all three sampling frequency. When we interpolated $9 / 19$ points to the $250 \mathrm{MHz}$ sampling chirps the positioning accuracy was better than both the $500 \mathrm{MHz}$ sampling frequency without interpolation points and the $1 \mathrm{GHz}$ sampling frequency without interpolation points. The accuracy of the $250 \mathrm{MHz}$ sampling frequency with $9 / 19$ interpolation points was close to that of the $2.5 \mathrm{GHz} / 5 \mathrm{GHz}$ sampling frequency without interpolation points. But when the interpolation points becomes more, the improvement of the positioning accuracy was not obvious. The positioning accuracy of $250 \mathrm{MHz}$ sampling frequency with 19 interpolation points was not better than the $500 \mathrm{MHz}$ sampling frequency with 9 interpolation points.

\section{CONCLUSION}

This paper introduced the present situation and future development of the small range wireless positioning, summarized the technologies and algorithms, and designed an improved positioning system which proposed the improved Sinc interpolation algorithm to reduce the sampling frequency of the system, calculated TODA values with matched filter, estimated the position of the target node by Chan's algorithm and used Chauvenet criterion to optimize the positioning results. Analysis and simulation results showed that we could reduce the sampling frequency to a great extent without reducing the accuracy in the improved positioning system. The accuracy could be about $1 \mathrm{~cm}$ in static condition and blow $10 \mathrm{~cm}$ in dynamic condition. So this improved system apparently had many advantages and high research value.

This proposed wireless positioning scheme also had stronger anti-interference ability and was easier to implement, which made it more promising and was worthy of continuous research. The system didn't need strict time synchronization between the transmitter and receivers, so it didn't need very sophisticated equipments and it was not costly compared to the existing methods. It could be used in many fields such as warehousing, parking lot, robot localization, environment monitoring, health care, construction, logistics, and so on. As the demands for small range wireless positioning grew, this system would have a very broad application prospects.

The problem was that the positioning algorithm used the improved Sinc interpolation algorithm and we had to wait for a certain amount of chirp to form the new chirp to calculate the TDOA values. And when there were more than one target nodes in the positioning area, there would be interference among the target nodes. The next work was to study multiple target nodes interference and proposed multiple target nodes coordination algorithms to confine the positioning delay and reduce and interference.

\section{ACKNOWLEDGMENT}

This work was supported by the Funding Project for Academic Human Resources Development in Institutions of Higher Learning under the Jurisdiction of Beijing Municipality under Grant PHR201007121, and Beijing Municipal Nature Science Foundation under Grant 4132026, and Beijing college student science research and entrepreneurial action plan (NO. 15006).

\section{REFERENCES}

[1] T. S. Rappaport, J. H. Reed, and B. D. Wonerner, "Position location using wireless communication on highways of thefuture," IEEE Communications Magazine, vol. 34, pp. 33-41, October 1996.

[2] S. Tekinary, E. Chao, and R. Richton, "Performance benchmarking for wireless location systems," IEEE Communications Magazine, vol. 36, pp. 72-76, 1998.

[3] H. Ahn and W. Yu, "Environmental-adaptive RSSI-based indoor localization," IEEE Trans. on Automation Science and Engineering, vol. 6, pp. 626-633, 2009.

[4] Y. Yu, Y. Yao, and X. Cheng, "TDOA positioning technology and practical application," China Radio, vol. 11, pp. 57-58, 2014.

[5] Z. Li, Z. Wang, Y. Zhang, and L. Ma, "Precision wireless positioning scheme in small range based on first-order difference and correlation inspection," Journal of Information Technology Research, vol. 6, pp. 1-15, July-Sept. 2013.

[6] Y. Jiang and T. Gu, "Finite length analysis of SinC interpolation," Chinese Journal of Scientific Instrument, vol. 26, pp. 987-990, Sept. 2005.

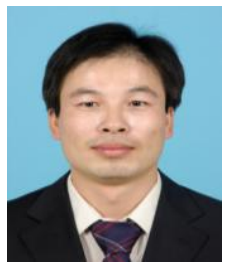

Zhengping Li received the doctor's degree in information and communication engineering at Beijing University of Posts and Telecommunications, Beijing, China, in 2008 .

$\mathrm{He}$ is now the associate professor in College of Information Engineering, North China University of Technology, Beijing. His primary role is as a tutor for the undergraduate and graduate students, cognitive 
radio systems, and network economics. He has vast experiences on the following technologies which include resource management in wireless networks, media access control, routing and QoS mechanism.

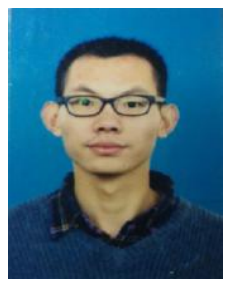

Chaoliang Qin is pursuing master degree in information and communication engineering at North China University of Technology, Beijing, China.

He works on various software development projects. His development skill set includes $\mathrm{C} / \mathrm{C}++$, Java and matlab. His major field of study includes wireless network positioning, signal and information processing and radio frequency identification tech.

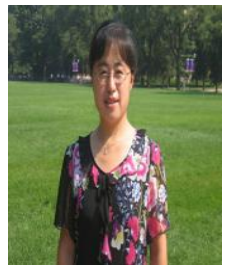

Yongmei Zhang worked as a post doctor in computer institute at Beijing University of Aeronautics and Astronautics, Beijing, China, in 2008.

She is now the professor in College of Information Engineering, North China University of Technology, Beijing. She has in-depth experiences on the following technologies which includes image processing, pattern recognition and artificial intelligence. She has over 10 years of experience in software development field.

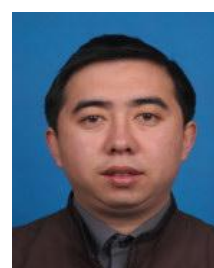

Li Ma received the doctor's degree in computer application technology at Beijing Institute of Technology, Beijing, China, in 2006.

$\mathrm{He}$ is now the professor in College of Information Engineering, North China University of Technology, Beijing. He has in-depth experiences on the following technologies which includes advanced computing technology, grid computing, multi-agent system and embedded system. He has over 10 years of experience in software development field.

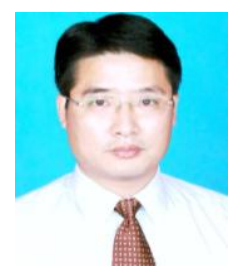

Changliu Niu received the doctor's degree in electromagnetic field and electromagnetic wave curriculum at Beijing University of Posts and Telecommunications, Beijing, China, in 2008.

$\mathrm{He}$ is now the associate professor in College of Information Engineering, North China University of Technology, Beijing. He has in-depth experiences on the following technologies which includes high speed optical communication, communication network and wireless sensor network. 